\title{
PREDICT Prostate, a useful tool in men with low- and intermediate-risk prostate cancer who are hesitant between conservative management and active treatment
}

\author{
Gaëtan Devos and Steven Joniau* (D
}

Keywords: Prostate cancer, Prognosis, Prostate cancer-specific mortality, Decision aid

\section{Background}

Risk stratification tools are useful to optimize treatment decisions in each individual patient, reducing both underand overtreatment. Currently, several such tools are available to estimate disease aggressiveness in patients with localized prostate cancer (PCa). These include nomograms (e.g., Memorial Sloan-Kettering Cancer Center (MSKCC) nomogram), tiered classification systems (e.g., D’Amico, National Comprehensive Cancer Network ( $\mathrm{NCCN})$ ), and risk assessment scoring systems (e.g., the cancer of the prostate risk assessment (CAPRA) score) [1]. Preoperative parameters such as biopsy Gleason score (or ISUP grade), initial PSA at time of biopsy, and clinical T-stage at digital rectal examination are used in these tools. The D'Amico classification, an easy-to-use three-tier classification system based on these three preoperative parameters, is adopted in daily clinical practice and endorsed by several international guidelines, such as the European Association of Urology (EAU) prostate cancer guidelines [2]. However, most of these risk stratification tools are designed to predict the risk of PSA recurrence after primary treatment rather than survival and do not take into account individual competing risk mortality in terms of age and comorbidity.

This comment refers to the article available at https://doi.org/10.1186/ s12916-020-01606-W.

* Correspondence: Steven.joniau@uzleuven.be

Department of Urology, University Hospitals Leuven, Leuven, Belgium

\section{PREDICT Prostate, a novel promising tool}

The PREDICT Prostate tool, on the other hand, is a multivariable prognostic model that provides individualized cancer-specific and overall long-term survival estimates in localized PCa patients [3]. In addition to the use of routinely available preoperative clinical-pathological variables such as PSA, biopsy Gleason score (ISUP grade group), and clinical T-stage, the PREDICT Prostate tool also includes the impact of patient characteristics (age and comorbidity status) and radical treatment (radical prostatectomy or radiotherapy) on survival. Thurtle et al. [4] performed an external validation of their previously published PREDICT Prostate model. Applied to the large Swedish PCBaSe cohort, the tool was able to discriminate patients who faced PCa-specific mortality and outperformed other widely used models such as the CAPRA and the three-tier EAU classification. It was proven to generally have high c-indices for all-cause and PCa-specific mortality, and the model calibration was good and remained accurate within the treatment subgroups.

However, some issues need to be emphasized before using the tool in daily clinical practice. First, the two cohorts (original United Kingdom (UK) and Swedish $\mathrm{PCBaSe}$ cohort) are generally very similar in epidemiological characteristics. Yet, more than half $(53 \%)$ of the patients in the PCBaSe had low-grade disease (ISUP grade group 1) compared to $32 \%$ in the original UK cohort. This high number of low-risk patients may affect the discriminatory power of the tool as these men are 
unlikely to die from their $\mathrm{PCa}$. Second, although reasonably high numbers of high-risk PCa patients (according to EAU risk grouping system) were included in the development and PCBaSe cohorts $(22.4 \%$ and $15.1 \%$ ISUP grade group 4-5 $\mathrm{PCa}$, respectively; $14.5 \%$ and $16 \%$ stage T3-4, respectively), it is unclear how well the model performs in actively treated high-risk and very-high-risk $\mathrm{PCa}$ patients. International guidelines recommend conducting multimodal therapy in high-risk $\mathrm{PCa}$ patients combining surgery, radiation therapy, and systemic therapy [5]. However, multimodal therapy was not considered radical therapy in these cohorts. In addition, the model assumes "equality" of surgery and radiotherapy as radical therapy. While this may be true for low-risk and some intermediate-risk PCa patients [6], this is far from certain in high-risk, locally advanced disease. In addition, a large proportion of the patients were treated with androgen deprivation therapy (ADT) alone (31.5\% in the original UK cohort and $23.1 \%$ in the external validation Swedish $\mathrm{PCBaSe}$ cohort). The $\mathrm{PCa}$ patients receiving ADT monotherapy tended to have high-risk localized $\mathrm{PCa}(71 \%$ and $78.8 \%$ in the original and PCBaSe cohort, respectively) or likely to have significant comorbidities excluding active local treatment. Approximately half of the high-risk patients in the original and external validation cohorts ( $47 \%$ and 52\%, respectively) received ADT monotherapy. Today, international PCa guidelines strongly recommend against the use of ADT monotherapy in newly diagnosed, non-metastatic PCa patients [2]. Therefore, the model is less useful in optimizing the therapy decision in this high-risk population. Third, the type of risk stratification tools that have been used to compare the prognostic value of the PREDICT model (NCCN and EAU) are risk grouping systems and are not developed to predict mortality. On the other hand, it would be of great importance to compare, for example, the PREDICT Prostate model with the nomogram proposed by MSKCC, which is similar [7]. In addition, the PREDICT Prostate model does not contain genomic tests or molecular markers. The combination of clinical and genomic biomarkers' tests (such as Decipher) has already been shown to improve the prognostic ability $[8,9]$. Ideally, the model should be validated using data from prospective, randomized trials such as the PROTECT dataset [6]. Furthermore, the comorbidity status in PREDICT Prostate is defined by "any hospital admission in the last 2 years prior to $\mathrm{PCa}$ diagnosis." While this allows clinicians to assess the impact of competing risks on the benefit of treatment, the implementation of more detailed comorbidity assessments such as the Charlson Comorbidity Index at the time of diagnosis will certainly improve the prognostic model [10]. Finally, the tool does not take into account recent changes in PCa management, such as the implementation of MRI prior to prostate biopsy or the use of targeted biopsies.

\section{Conclusions}

Nevertheless, the results of the external validation are impressive and of great importance to the uro-oncological community to optimize treatment decisions in localized PCa patients. By including the impact of radical treatment on survival, the tool is especially useful in men who are hesitant between active surveillance or active treatment within their own context of competing mortality. However, the utility of the PREDICT Prostate tool in high-risk PCa patients is questionable.

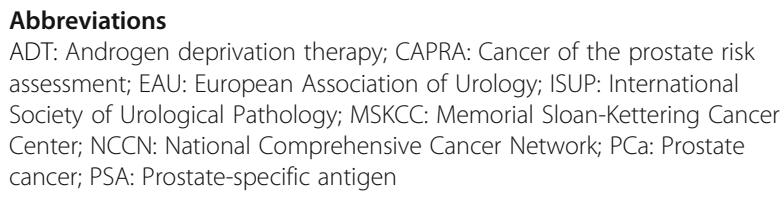

Authors' contributions

The authors read and approved the final manuscript.

Funding

Not applicable

Availability of data and materials

Not applicable

Ethics approval and consent to participate

Not applicable

Consent for publication

Not applicable

Competing interests

None to declare

Received: 25 June 2020 Accepted: 25 June 2020

Published online: 16 July 2020

\section{References}

1. Zelic R, Garmo H, Zugna D, Stattin P, Richiardi L, Akre O, et al. Predicting prostate cancer death with different pretreatment risk stratification tools: a head-to-head comparison in a nationwide cohort study. Eur Urol. 2020; 77(2):180-8.

2. Mottet N, Bellmunt J, Bolla M, Briers E, Cumberbatch MG, De Santis M, et al. EAU-ESTRO-SIOG guidelines on prostate cancer. Part 1: screening, diagnosis, and local treatment with curative intent. Eur Urol. 2017;71:618-29.

3. Thurtle DR, Greenberg DC, Lee LS, Huang HH, Pharoah PD, Gnanapragasam VJ. Individual prognosis at diagnosis in nonmetastatic prostate cancer: Development and external validation of the PREDICT Prostate multivariable model. PLoS Med. 2019;16(3):e1002758. https://doi.org/10.1371/journal. pmed. 1002758.

4. Thurtle D, Bratt O, Stattin P, et al. Comparative performance and external validation of the multivariable PREDICT Prostate tool for non-metastatic prostate cancer: a study in 69,206 men from Prostate Cancer data Base Sweden (PCBaSe). BMC Med. 2020;18:139 https://doi.org/10.1186/s12916020-01606-W.

5. Moris L, Cumberbatch MG, Van den Broeck T, Gandaglia G, Fossati N, Kelly $B$, et al. Benefits and risks of primary treatments for high-risk localized and locally advanced prostate cancer: an international multidisciplinary systematic review. European Urology. Elsevier B.V.; 2020. 
6. Hamdy FC, Donovan JL, Lane JA, Mason M, Metcalfe C, Holding P, et al. 10year outcomes after monitoring, surgery, or radiotherapy for localized prostate cancer. N Engl J Med. 2016;375(15):1415-24.

7. Prostate cancer nomograms: dynamic prostate cancer nomogram: coefficients | Memorial Sloan Kettering Cancer Center. [cited 2020 Jun 16]. Available from: https://www.mskcc.org/nomograms/prostate/pre_op/ coefficients.

8. Cooperberg MR, Davicioni E, Crisan A, Jenkins RB, Ghadessi M, Karnes RJ Combined value of validated clinical and genomic risk stratification tools for predicting prostate cancer mortality in a high-risk prostatectomy cohort. Eur Urol. 2015;67(2):326-33.

9. Spratt DE, Zhang J, Santiago-Ji enez M, Dess RT, Davis JW, Den RB, et al. Development and validation of a novel integrated clinical-genomic risk group classification for localized prostate cancer. J Clin Oncol. 2018;36(6): 581-90.

10. Charlson ME, Pompei P, Ales KL, MacKenzie CR. A new method of classifying prognostic comorbidity in longitudinal studies: development and validation. J Chronic Dis. 1987;40(5):373-83.

\section{Publisher's Note}

Springer Nature remains neutral with regard to jurisdictional claims in published maps and institutional affiliations.

- fast, convenient online submission

- thorough peer review by experienced researchers in your field

- rapid publication on acceptance

- support for research data, including large and complex data types

- gold Open Access which fosters wider collaboration and increased citations

- maximum visibility for your research: over $100 \mathrm{M}$ website views per year

At $\mathrm{BMC}$, research is always in progress. 